\title{
Separation of Some Anionic Dyes Using Reverse Micelles of CTAB and SDS as Efficient Surfactants Adsorbents from Aqueous Medium
}

\author{
Heba M. El Refay (D, Nagwa Badawy, Amina Hamada, and El Shimaa Hassen \\ Al-Azhar University (Girls), Faculty of Science (Girls), Department of Chemistry, Yousef Abbas Str., Nasr City, Cairo, Egypt \\ Correspondence should be addressed to Heba M. El Refay; hebaelrefay.5919@azhar.edu.eg
}

Received 14 September 2021; Accepted 5 December 2021; Published 17 January 2022

Academic Editor: Sébastien Déon

Copyright (c) 2022 Heba M. El Refay et al. This is an open access article distributed under the Creative Commons Attribution License, which permits unrestricted use, distribution, and reproduction in any medium, provided the original work is properly cited.

\begin{abstract}
Extractive removal of anionic dyes, namely, Color Index (CI) Reactive Blue 222 and Reactive Yellow 145, using reverse micelles based on liquid-liquid extraction (LLE) was carried out from aqueous solutions using different anionic and cationic surfactants (e.g., sodium dodecyl sulfate (SDS) and cetyltrimethylammonium bromide (CTAB), resp.), which dissolved in ethyl acetate as solvent. The reverse micelle principal acts on the dye encapsulated in the solvent in an aqueous micropool. The experiments were carried out by mixing in a simple mixer a given amount of dyes and surfactants dissolved in a solvent in an aqueous process. Due to gravity, the dye is separated from water after the solvent phase is separated from the aqueous phase, including dye encapsulated in reverse micelles. Under various experimental conditions, extraction efficiency was studied, including solution $\mathrm{pH}$, extraction time, initial dye concentration, extractant concentration, temperature, stripping agent, and solvent reusability. Dyes extracted were stripped quantitatively with $\mathrm{NaOH}$ solution. Recovery of the solvent and the reuse of dyes and surfactants after extraction of dye molecules from reverse micelles surfactant core considered are very important from an economic point of view. The optimized conditions were $7 \pm 0.2$ solution $\mathrm{pH}, 9 \times 10^{-2} \mathrm{~mol} / \mathrm{L}$ extractant concentration, $1 \mathrm{M} \mathrm{NaOH}$ stripping agent concentration, $60 \mathrm{~min}$ extraction time, $6 \times 10^{-5} \mathrm{~mol} / \mathrm{L}$ dye concentration, and $1: 1$ aqueous to organic $(\mathrm{A} / \mathrm{O})$ ratio. $87-93 \%$ of dyes were extracted at experimental optimum conditions.
\end{abstract}

\section{Introduction}

Dyes are organic compound and highly colored, so the extract of colors from aqueous environments is one of the most challenging criteria faced by the manufacture of dye, resulting in significant environmental problems. Numerous researchers have studied various techniques, including micellar enhanced ultrafiltration, [1] adsorption, [2] flocculation [3], and precipitation [4], for removal of colored dyes from aqueous environments. All these mentioned techniques have been contrasted, but each showed both advantages and disadvantages. The organic contaminants negatively affect human health causing cancer, congenital abnormalities, and immune and reproductive system dysfunction. They also affect the growth of infants and children [5]. Also, the discharge of water from the textile, chemical, fertilizer, pesticide, metal, battery, and paper industries contains many types of organic pollutants (dyes, pharmaceutical ingredients, pesticides, organic halides, fertilizers, phenols, and surfactants) that should be extracted before they are released into the environment to prevent health risks and avoid destroying the ecosystem [6-8].

The extraction of dye contamination from aqueous environments using LLE process, resulting from reverse of micelle extraction (RME), is the focus of this study. The LLE process has been utilized as a treating method for separating organic analytes from aqueous samples and isolating them [9]. LLE method is commonly used for sample preparation and depends on the analytes transfer from an aqueous phase to water-immiscible solvent $[9,10]$.

The organic pollutants are separated from aqueous environments due to the effect of surfactants, using low- 
solubility compounds that have been solubilized in the organic solvent by reverse micelles [11]. RME occurs when a substrate in water-immiscible solvent is extracted from aqueous phase to the organic phase by reverse micelles as shown in Figure 1 [12]. Ethyl acetate, also known as acetic acid ethyl ester, acetic ester, and acetic ether, has a relative density of $0.902 \mathrm{~g} / \mathrm{cm}^{3}$ and has poor water solubility. Among the solvents, ethyl acetate is favored since it has demonstrated a quicker separation of kinetics from the aqueous phase.

The surfactants are a group of organic compounds with a hydrophilic head and a hydrophobic tail (hence known as amphiphiles). The head part shows the hydrophobic nature and tail shows hydrophilic nature. With the increase in the concentration of surfactant, amphiphiles start aggregating in the bulk to form aggregates. The concentration at which this occurs is known as critical micelle concentration (CMC) with increase in concentration of surfactants causing an increase in the concentration of reverse micelles resulting in an increase in the extraction of dye from the aqueous media, due to electrostatic attraction between the oppositely charged dye and surfactants molecules. The surfactant option should be based on the hydrophilic group's properties to facilitate electrostatic interaction of attractive separation of the surfactant from the dye [13]. The surfactants most widely used in the extraction process are SDS and CTAB [14]. Some physical properties of such surfactants are shown in Table 1 [15-17]. The surfactant reverse micelles in recent years were used to remove and separate the dyes by extraction from aqueous solution, since they are helpful in terms of water conservation, cost reduction, and being environmentally friendly [18]. More significantly, it is possible to reuse the solvents and recycle the dyes from water to fabric dyeing.

The mechanism of extraction has three regions: (a) an external zone in which the tails of hydrophobic surfactant are in contact with the hydrophobic solvent through forces of dispersing, (b) a region where molecules of water reacted with the head of the surfactant's hydrophilicity through iondipole, dipole-dipole, and hydrogen bond interactions, and (c) the center of a micellar which is a water cage that is relatively thin. The method of water purification occurs as reverse micelles in inner region are more capable of solubilizing the polar molecules and ionic compounds in contrast to the initial aqueous solution $[19,20]$. Three characteristics basically rely on the efficiency of the RME technique, the surfactants, the organic solvent, and the ionic residual in the water. The conditions that must be met for the organic solvent are that it must be low in cost, low in volatility, low in toxicity, and of easy reusability $[12,21,22]$.

The objective of this research is to see if reverse micelles can be used to separate organic dyes from water using liquidliquid extraction. As seen in Figure 2, the two dyes chosen, Reactive Blue 222 and Reactive Yellow 145, are anionic in nature. They were removed, and a reverse micelles ionic counter was utilized to recover the dyes that were good in dyeing cotton cloth. The effect of variables such as surfactants, dye concentrations, solution $\mathrm{pH}$, stripping agent, contact time, and temperature on dye extraction efficiency from aqueous solution was investigated.
The application of the separation method to the process of removing polluting organic ions from wastewater, rather than the most common adsorption process in current research, is novel in this study, and the separation process is characterized by ease of implementation, like the adsorption process.

\section{Materials and Methods}

2.1. Materials. In this study, all the chemical materials used in these experiments were reagents of analytical grade. Surfactants (anionic as SDS and cationic as cetyltrimethylammonium bromide, CTAB (SD Fine Chem. Ltd., purity $\left.{ }^{>} 95.0 \%\right)$ ) and solvents used for extracting dye from aqueous media were ethyl acetate. Sodium hydroxide, hydrochloric acid, and sulfuric acid as stripping agents were supplied from Sigma Aldrich, Cairo, Egypt. The dyes used in this research are CI Reactive Blue 222 (molecular formula $\mathrm{C}_{37} \mathrm{H}_{23} \mathrm{C}_{1} \mathrm{~N}_{10} \mathrm{Na}_{6} \mathrm{O}_{22} \mathrm{~S}_{7}$ ) and CI Reactive Yellow 145 (molecular formula $\mathrm{C}_{28} \mathrm{H}_{20} \mathrm{C}_{1} \mathrm{~N}_{9} \mathrm{Na}_{4} \mathrm{O}_{16} \mathrm{~S}_{5}$ ). They were supplied from Imperial Chemical Industries, ICI. For solution preparation and dilution, bidistilled water was utilized in the experiments. All chemicals were used without further purifications. The dye absorbance and concentration were measured using UV-visible spectrophotometer (Model T60 Spectrophotometer, United Kingdom). The $\mathrm{pH}$ meter (WTW, Germany) was used to measure the solution $\mathrm{pH}$, and the sample shaker (IKA-KS 501, Germany) was used for solutions agitation.

2.2. Synthetic Dye Solution Preparation. The stock solutions of anionic dyes (C.I. RB222 and C.I. RY145) were prepared by weight of the dried dye in doubly distilled water by concentration of $1 \times 10^{-2} \mathrm{~mol} / \mathrm{L}$. Both dyes are highly water soluble.

2.3. Experimental Procedure. Figures 3 and 4 present a schematic representation of the experimental method. The magnetic simple stirrer was used for mixing the dye in the aqueous phase and the solvent. The stirrer speed can be reliably measured (rpm). To separate the solvent organic and aqueous phases, a separating funnel was used by gravity. For measuring the intensity of dye color before and after extraction from aqueous media, a UV spectrophotometer (Model T60 Spectrophotometer) was used.

2.4. Method of Extracting Dye. Extraction of both RB222 and RY145 dyes using reverse micelles by LLE method was performed in two steps. In the first step, $10 \mathrm{ml}$ of ethyl acetate as a solvent containing a given amount from $\mathrm{CTAB}$ or SDS surfactants was mixed with $10 \mathrm{ml}$ of a given concentration of dye prepared $\left(3-15 \times 10^{-5} \mathrm{~mol} / 10 \mathrm{ml}\right)$. Surfactant concentrations were around the essential micelle concentration (CMC) level for all surfactants. The concentrations of surfactants used in this study ranged between $9 \times 10^{-3}, 1,3,5,7$, and $9 \times 10^{-2} \mathrm{~mm} / \mathrm{L}$. A magnetic stirrer was used to mix the aqueous phase and ethyl acetate at $100 \mathrm{rpm}$ 


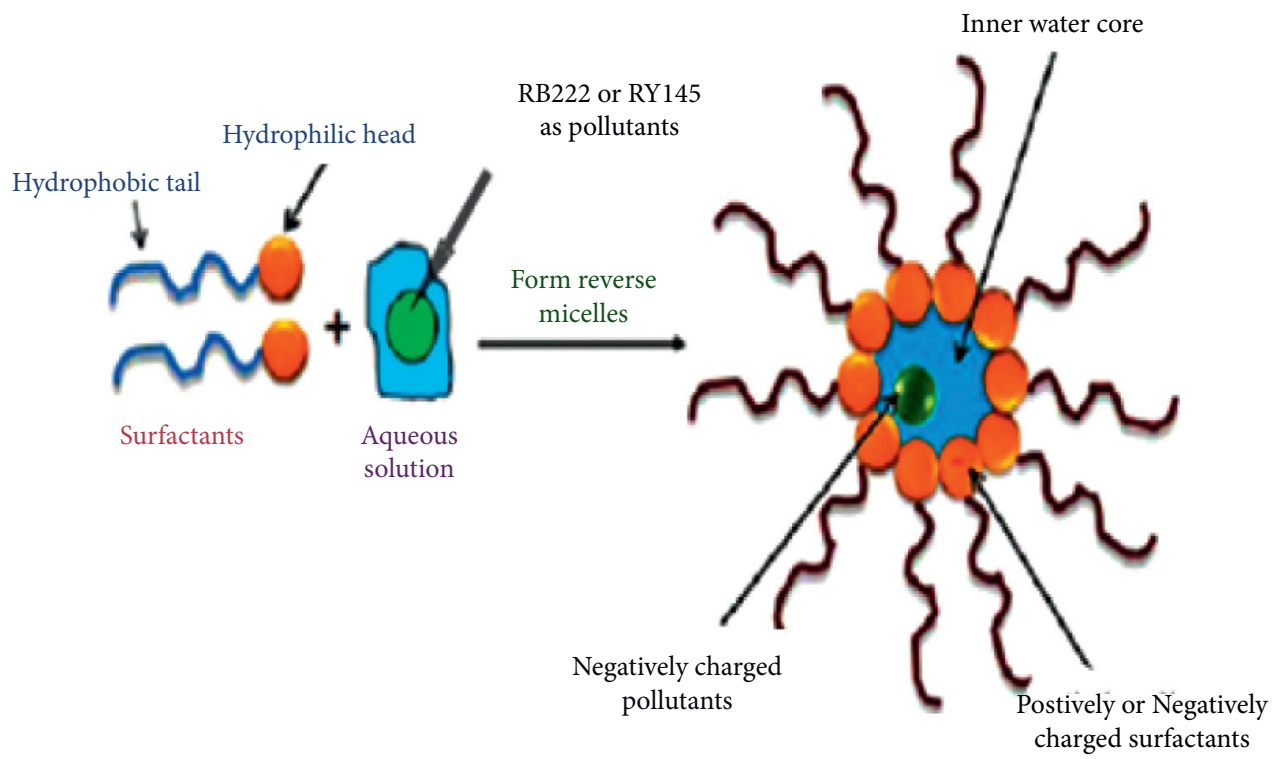

FIgURE 1: Schematic drawing using reverse micelles for solvent extraction [22].

TABle 1: Physical properties of surfactants.

\begin{tabular}{lcc}
\hline Name & Cetyltrimethylammonium bromide (CTAB) & Sodium dodecyl sulfate (SDS) \\
\hline Molecular formula & {$\left[\left(\mathrm{C}_{16} \mathrm{H}_{33}\right) \mathrm{N}\left(\mathrm{CH}_{3}\right)_{3}\right] \mathrm{Br}$} & {$\left[\mathrm{C}_{12} \mathrm{H}_{25} \mathrm{SO}_{4}^{-} \mathrm{Na}^{+}\right]$} \\
Molecular weight & 364.45 & 288.37 \\
Types & Cationic & Anionic \\
Appearance & White & Light yellow \\
CMC & Lies in the range $0.88-1.5 \mathrm{mM}$ (in water at $\left.25^{\circ} \mathrm{C}\right)[15,16]$ & $8.2 \mathrm{mM}$ (in water at $\left.25^{\circ} \mathrm{C}\right)[17,23]$ \\
\hline
\end{tabular}

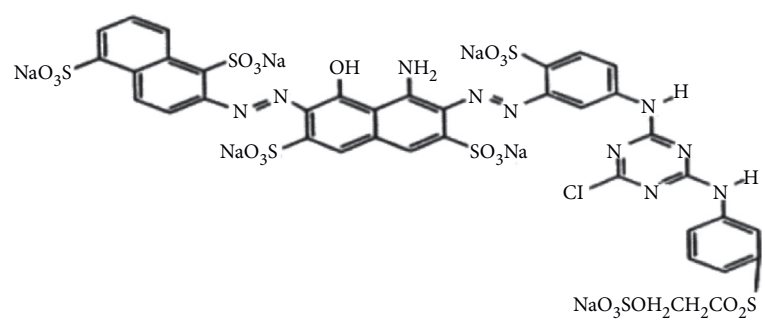

(a)

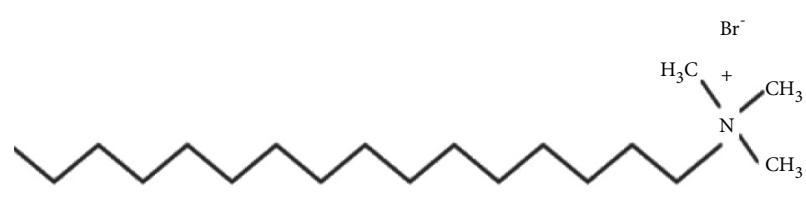

(c)

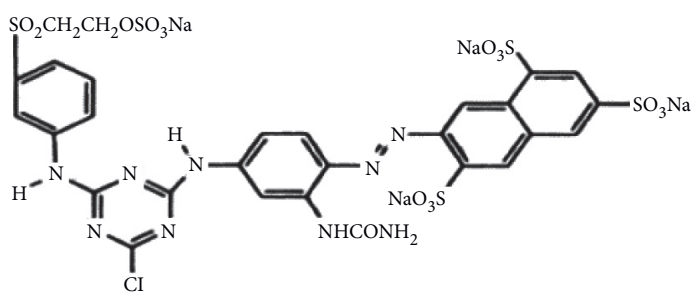

(b)

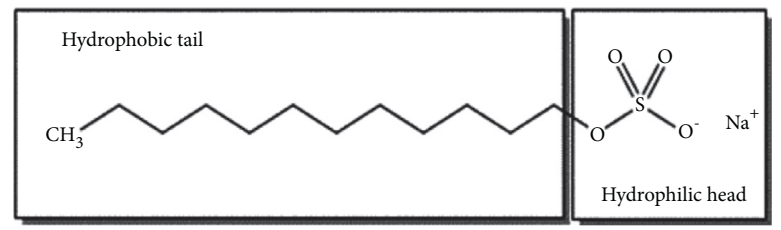

(d)

FIgURE 2: Chemical structures of ionic dyes (a) RB222, (b) RY145, (c) CTAB, and (d) SDS as surfactants.

for 5 minutes at $25^{\circ} \mathrm{C}$. A whole solution dispersion was moved to the separating funnel in the next stage for gravitationally separating the organic solvent from the aqueous phase from the previous step, leading to the formation of two transparent liquid media. The heavier aqueous medium was collected at the bottom of a separating funnel, while the lighter ethyl acetate containing encapsulated dye in the reverse micelles was collected at the top. The two dispersion phases were permitted to stay about 24 hours, in order to achieve full separation of the pure phase. Experiments have been carried out at $25 \pm 1^{\circ} \mathrm{C}$ for $24 \mathrm{~h}$ with $\mathrm{pH}(7.17 \pm 0.5)$; then the concentration of dye was measured. The amount of dyes separated which were present in the organic solvent was determined and analyzed through the use of UV spectrophotometer. The study of absorbency spectra showed the highest absorbencies for both RB222 and RY145 dyes at 620 and $423 \mathrm{~nm}$, respectively. The impact of the solution $\mathrm{pH}$ on the extracted dyes was studied. $0.5 \mathrm{~N} \mathrm{HCl}$ and $0.5 \mathrm{~N} \mathrm{NaOH}$ 


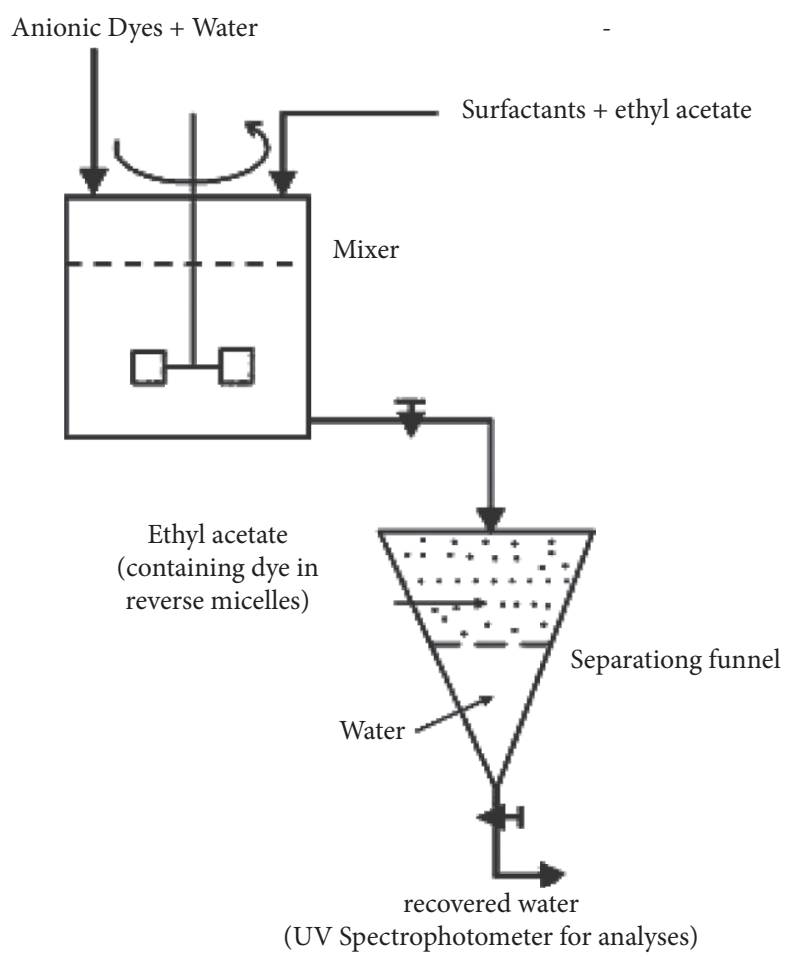

FIgURE 3: Experimental setup schematic diagram for the removal of dye from the aqueous phase [24].

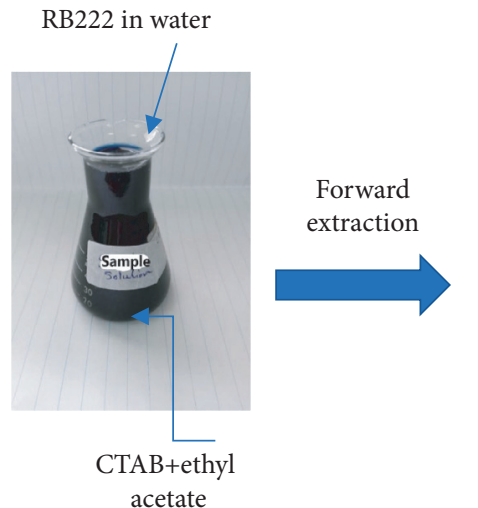

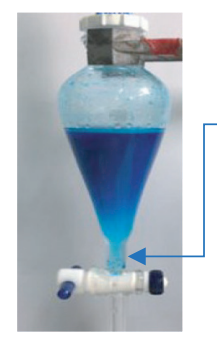

Separating funnel
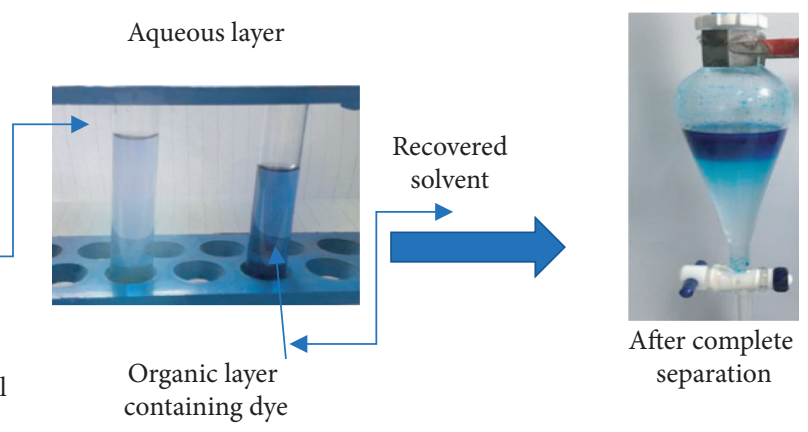

Figure 4: The experimental method for separation of RB222 dye from aqueous media and the extraction of solvent.

solutions were used to change the $\mathrm{pH}$ values from 2 to 10 . The extraction efficiency $(E)$ and the partition coefficient $(K)$ for dyes were calculated and given by the following equations $[12,25]$ :

$$
\begin{aligned}
\text { Extraction } \% & =\left(\frac{C_{i}-C_{a q}}{C_{i}} \times 100\right), \\
K & =\frac{C_{\text {org }}}{C_{a q}},
\end{aligned}
$$

where $C_{i}, C_{a q}$, and $C_{\text {org }}$ are the initial dye concentration taken before extraction at $t_{0}$, the residual dye concentration in aqueous phase at $25 \mathrm{~h}$ after separating two layers, and dye concentration in organic phase encapsulated in the reverse micelles, respectively.
2.5. Stripping Process for Organic Solvent Recovery. After complete extraction of both RB222 and RY145 dyes from water using SDS and CTAB which were dissolved in ethyl acetate, the organic solvent was extracted and removed from aqueous phase. There were surfactant reverse micelles encapsulating dye in organic solvent of ethyl acetate, and the obtained water was pure from dyes. The phase of organic solvent was treated with $10 \mathrm{ml}$ stripping agent as $\mathrm{HCl}$ or $\mathrm{H}_{2} \mathrm{SO}_{4}$ or $\mathrm{NaO}$. By using the simple magnetic stirrer at $100 \mathrm{rpm}$ for $5 \mathrm{~min}$ at $25^{\circ} \mathrm{C}$, all solutions were mixed, and the layers were allowed to settle. A phase separation occurs at the top of the separation funnel with the acid/dye phase (without both surfactant and ethyl acetate). Ethyl acetate (recovered) was neutralized at $\mathrm{pH} \sim 7$ and reused for the treatment of fresh samples of colored water. Among the cycles, surfactant additions were required for the existence of surfactants to be 
recomposed. The efficiency of recovery (ER\%) of ethyl acetate was determined after the separation of RB222 and RY145 from the organic solvent. It was calculated by the following equation [12]:

$$
\mathbf{E R} \%=\left(\frac{C_{i}-C_{a q}}{C_{i}} \times 100\right),
$$

where $C_{i}$ is the concentration of either RB222 or RY145 dyes in the phase of ethyl prior to the addition of stripping agent $\left(t_{0}\right)$ and $C_{a q}$ is the concentration of either RB222 or RY145 dyes after the addition of stripping agent in the phase of ethyl acetate.

\section{Results and Discussion}

3.1. Extraction of RB222 and RY145. Extraction of the dyes from the aqueous medium was studied because they greatly affect the environment. The formation of reverse micelles of surfactants in the organic phase with dyes entrapment in its center resulted in the extraction of RB222 and RY145 by surfactants as shown in Figure 1. The size compatibility of the dye with the size of the reverse micelle's center is important for efficient dye extraction. When using the cationic CTAB to shape reversed micelles in the solvent of ethyl acetate, the removal of negatively charged RB222 and RY145 was favored. The molecules of dye are encapsulated inside the center and around the reversed micelles produced by head group of cationic $C T A B$ during stirring of the organic solvent and the aqueous phase mixture because the negative charges of RB222 and RY145 were attracted electrostatically to the head of the cationic CTAB group, in agreement with literature [26]. Back dye extraction from ethyl acetate into water was successfully accomplished by applying a counter ionic surfactant to the water. The formation of reverse micelles in ethyl acetate is supported by all this evidence.

The following sections discuss the impact of different variables on the percentage of dye extracted.

3.1.1. Interaction of LLE Variables on the Extraction Percentage of RB222 and RY145 from Aqueous Medium by Using Reverse Micelles of CTAB and SDS as Efficient Surfactants Adsorbents. The results were obtained from 3D experimental runs conducted to observe the effect of the four LLE factors (solution $\mathrm{pH}$, initial dye concentration, surfactants concentration, and time) using CTAB and SDS. The analyses were supported by the $3 \mathrm{D}$ plots for interaction influence of four factors on extraction percentage of both RB222 and RY145 dyes from aqueous medium using reverse micelles.

(1) The Interaction Influence of the Solution $p H$ and Initial Concentration of Dye. The interaction impact of the solution $\mathrm{pH}$ and concentration of dyes is represented in Figures 5(a)$5(\mathrm{~d})$, indicating that high dye concentration causes dye extraction to decrease, while the increasing $\mathrm{pH}$ causes dye extraction to increase. This may be due to the positive charges on the molecules of dye in acidic medium. From Figure 5, it can be seen that, at low $\mathrm{pH}$ value $(\mathrm{pH}<5.2)$, more than $62 \%$ and $72 \%$ of RY145 and RB222, respectively, were extracted by SDS surfactant, and ( $\mathrm{pH}<8.2)$ more than $94 \%$ and $85 \%$ of RY145 and RB222, respectively, were extracted by CTAB surfactant. Meanwhile, the increase in the solution $\mathrm{pH}(\mathrm{pH}<5.2$ or $<8.2)$ and the increase in the initial concentration of dye resulted in a substantial reduction in extraction percentage in agreement with literature [27]. The existence of such positively charged functional groups will attract the negatively charged dye ions; it results in an increase in the extraction rate of dyes from aqueous phase, and the tendency will reverse when the dye separation decreases as the concentration increases. This performance can be explained by the fact that increasing the concentration of adsorbate while keeping the surfactant concentration constant causes saturation of the reverse micelles, resulting in a drop in the adsorbate extract. Since reverse micelles have a constant capacity to encapsulate dye molecules, the number of these micelles was insufficient to contain the increased concentration of dyes, so the extraction percentage of both dyes decreases as the concentration of dyes increases at a fixed surfactant concentration.

(2) The Interaction Impact of Solution $\mathrm{pH}$ and Surfactants Concentration. Impact of the solution $\mathrm{pH}$ and surfactants concentration on RY145 and RB222 dye extraction by using reverse micelles of CTAB and SDS is shown in 3D plots in Figures 6(a)-6(d). Percent extraction increased with increasing adsorbent dose and increasing solution $\mathrm{pH}$; at lower $\mathrm{pH}(\mathrm{pH}<5.2)$, more than $62 \%$ and $72 \%$ of RY145 and RB222, respectively, were removed by SDS surfactant, and $(\mathrm{pH}<8.2)$ more than $94 \%$ and $85 \%$ of RY145 and RB222, respectively, were removed by CTAB surfactant. The maximum percentage extraction was predicated at solution $\mathrm{pH}<5.2$ or $<8.2$. Such behavior was justified by the presence of large quantity of positively charged surface functional groups at lower solution $\mathrm{pH}$ and large amount of surfactants concentration of $9 \times 10^{-2} \mathrm{~mol} / 10 \mathrm{ml}$ ethyl acetate for such a given dye concentration. With an increase in the concentration of CTAB and SDS surfactants from $9 \times 10^{-3}$ to $9 \times 10^{-2} \mathrm{~mol} / 10 \mathrm{ml}$ ethyl acetate for a given dye concentration, the extraction percentage of RB222 and RY145 from the aqueous media will be increased, due to the presence of the force attraction between dyes molecules and ionic surfactants which, in the solvent phase, was induced to the extraction of dyes from the aqueous media. At a known concentration of surfactant, ethyl acetate has a constant capacity to encapsulate the molecules of dye, due to the formation of constant number from reverse micelles. Thus, adding surfactants resulted in increasing the reverse micelles number according to the thermodynamic model based on mass action kinetics and increasing solubilization with increasing the concentration of surfactant explained by this model, where molecule of dye is $D$, attracting with $n$ reverse micelles empty $M$, to form DM complex, which is reverse micelle filled by dye molecules, according to the pseudoreaction [25]:

$$
D+n M=\mathrm{DM}+[(n-1) M] .
$$




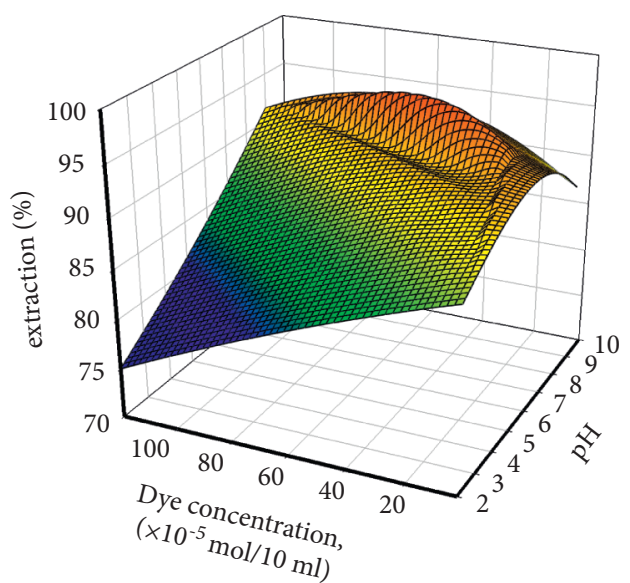

(a)

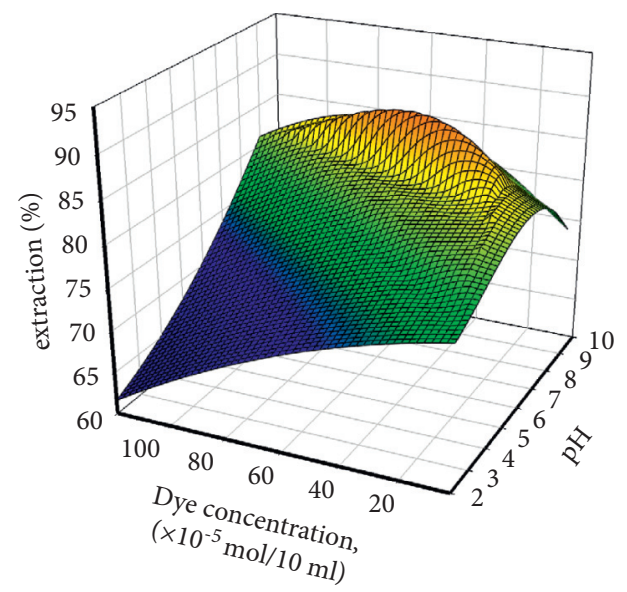

(c)

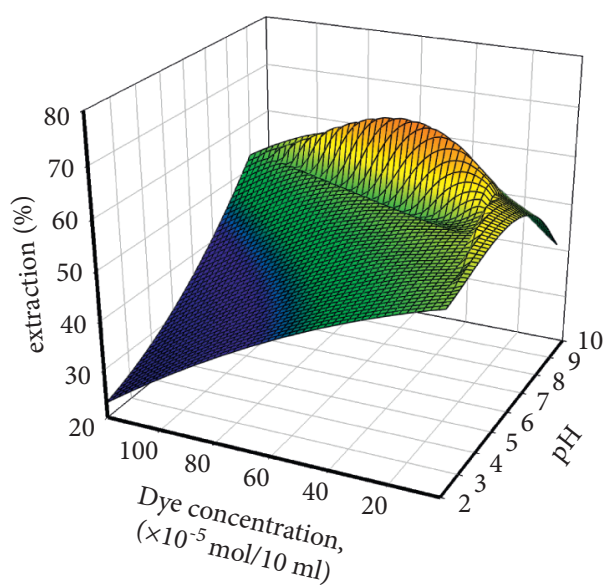

(b)

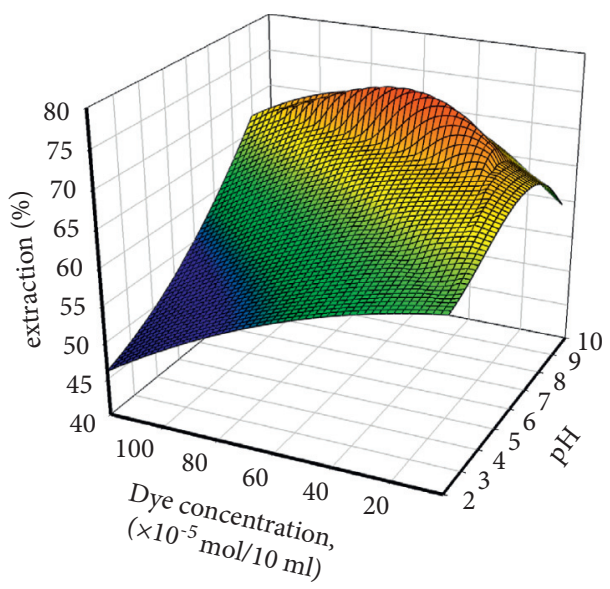

(d)

FIGURE 5: 3D plots of the interaction influence of solution $\mathrm{pH}$ and the initial concentration of RY145 dye on extraction\% by (a) CTAB and (b) SDS and RB222 dye by (c) CTAB and (d) SDS.

The increase in the concentration of surfactants $[S]$ caused an increase in the concentration of reverse micelles $[M]$, resulting in an increase in the extraction of dye from the aqueous media, due to coulombic attraction between the dye molecules and surfactants. It should be remembered that the extraction of the dyes is achieved by electrostatically attracting the oppositely charged dye and surfactant molecules so RB222 and RY145 were extracted by attraction coulombic between their anionic group $\left(-\mathrm{SO}_{3}^{-}\right)$and cationic head group $\left(-\mathrm{N}^{+} \mathrm{R}_{3}\right)$ of CTAB, while RB222 and RY145 were extracted by a comparable extraction by the attraction forces between positive $\left(-\mathrm{N}^{+}\right.$charge $)$and anionic SDS $\left(-\mathrm{SO}_{3}^{-}\right)$. Such trends were also observed in the previous study [25]. Thus, it is easier for RB222 and RY145 to be separated by CTAB from aqueous phase to solvent phase. If very low concentrations of surfactants are used, the separation of dye from the water phase is very low.

(3) The Interaction Influence of Solution $p H$ and Contact Time. 3D surface plots for combined solution $\mathrm{pH}$ and contact time effect are shown in Figures 7(a)-7(d). At $\mathrm{pH}$ $<5.2$ or $<8.2$, for RY145 and RB222 using SDS and CTAB, with increasing contact time, the extraction of dyes increases; as LLE time increases, the dye extraction\% increases. This result indicates that LLE factors have a significant effect on the extraction\% of dye [23].

(4) The Interaction Effect of Surfactants Concentration and Concentration of Dye. Interaction impact of surfactants concentration and the initial concentration of dye on the extraction of dyes in $3 \mathrm{D}$ surface plots is shown in Figures $8(a)-8(d)$. It is observed that, with increasing concentration of surfactants and decreasing concentration of the RY145 and RB222, the extraction of both RY145 and RB222 dyes increased. This is due to the fixed capacity of ethyl acetate to encapsulate molecules of dye for a known concentration of surfactant, with fixed capacity of reverse micelles. Dye extraction percentage was smaller as the constant number of reverse micelles was not sufficient to cover the increase of dye molecules. So, the increase in the concentration of dye leads to a decrease in the extraction\% [27].

(5) The Interaction Influence of the Initial Concentration of Dye and Contact Time. In Figures 9(a)-9(d), 3D surfaces plots display the interactive impact of the concentration for 


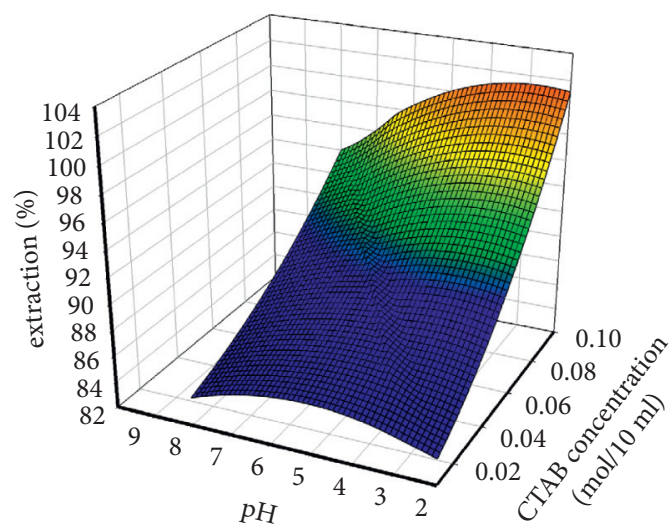

(a)

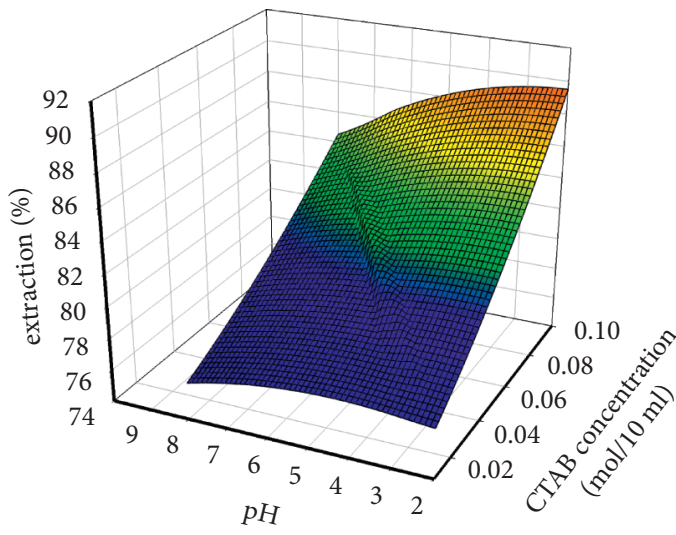

(c)

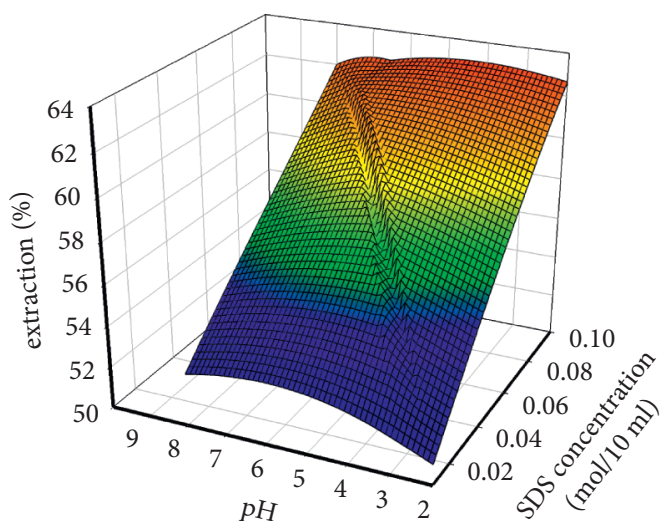

(b)

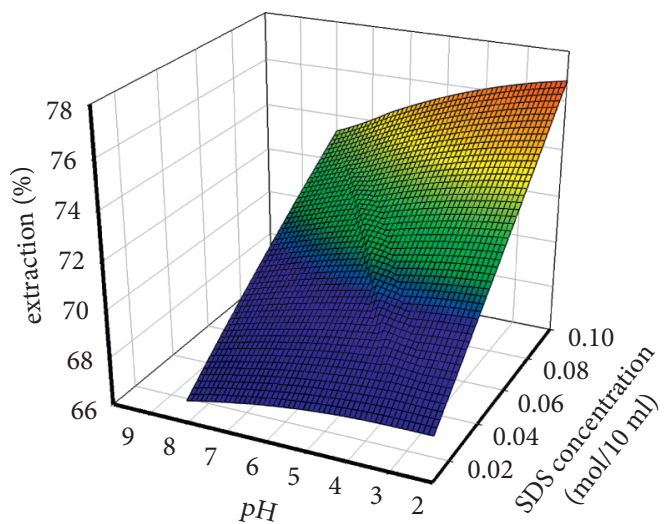

(d)

FIGURE 6: 3D plots of the interaction influence of solution $\mathrm{pH}$ and adsorbent dose on the extraction\% of RY145 by (a) CTAB and (b) SDS and RB222 by (c) CTAB and (d) SDS.

RY145 and RB222 dyes and contact time on extraction of dye. At 50 minutes, the higher amount of RY145 dye was extracted, and at 60 minutes, the RB222 dye was extracted. After this time, the efficiency of extraction remained constant. The equilibration time for further studies was set at 50 and 60 minutes for RY145 and RB222, respectively, increasing the contact time with no considerable improvement in dyes extraction\%, in agreement with literature [24].

(6) The Interaction Effect of Surfactants Concentration and Contact Time. Figures 10(a)-10(d) illustrate the combination effect of CTAB or SDS surfactants concentration and contact time on extraction of RY145 and RB222 dyes from aqueous media using reverse micelles. It is obvious that as the surfactants concentration and contact of time increased, the extraction of both dyes increased. This is because an increase in the concentration of surfactants would make higher numbers of reverse micelles available $[19,25]$.

3.2. Effect of Temperature. As shown in Figures 11 and 12, the influence of the temperature on the extraction of both RY145 and RB222 dyes from aqueous media was studied. These figures clearly indicate that the extraction percentage of both dyes increased when the temperature was raised from 25 to $50^{\circ} \mathrm{C}$. The degree of dyes solubilization increases at higher temperatures, and the rise in the temperature contributes to an increase in the micelles number aggregated in agreement with literature [28]. Therefore, the temperature has no significant effect on the extraction of the dye from aqueous media at room temperature. Increasing the extraction efficiency or dyes solubilization in micelles with the rise in temperature occurs by reducing the polarity of RY 145 and RB222 molecules as temperature increases which indicates a preferentially hydrophobic nature. So, in the micelles, RY145 and RB222 molecules get solubilized. This is improved at higher temperatures by an increase in the number of aggregations, which contributes to increasing the efficiency of extraction.

\subsection{Effect of LLE Parameters on the Partition Coefficient of RB222 and RY145 from Aqueous Medium Using Reverse Micelles Surfactants as Adsorbents}

3.3.1. Effect of Dyes Concentration. Influence of dye concentration on the partition coefficient $(K)$ as shown in Figure 13 of RY145 and RB222 dyes was studied, with increasing the concentration of both dyes from $(3-5) \times$ 


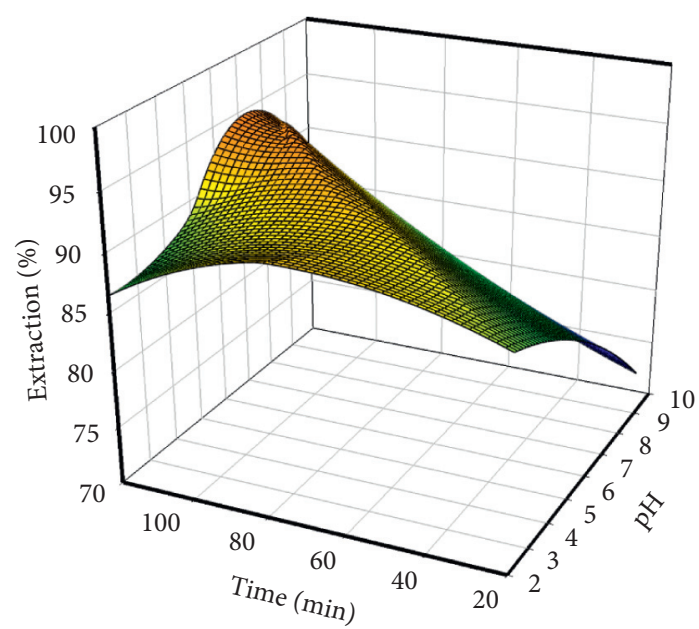

(a)

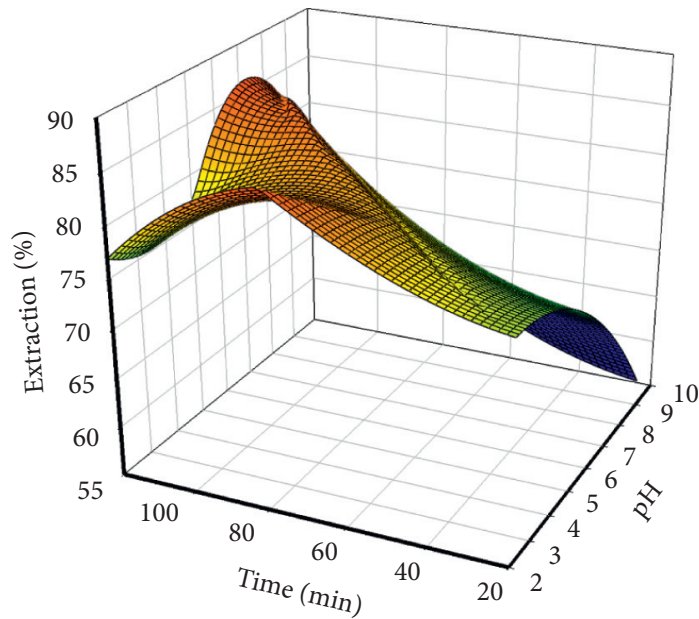

(c)

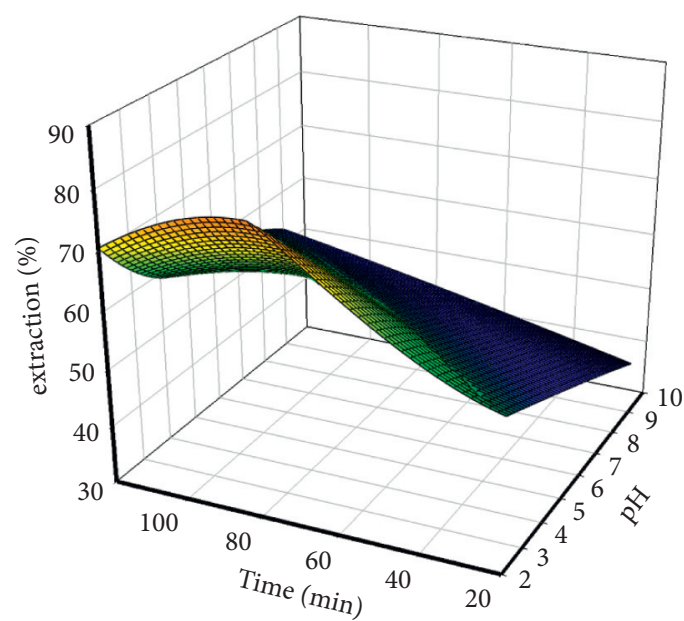

(b)

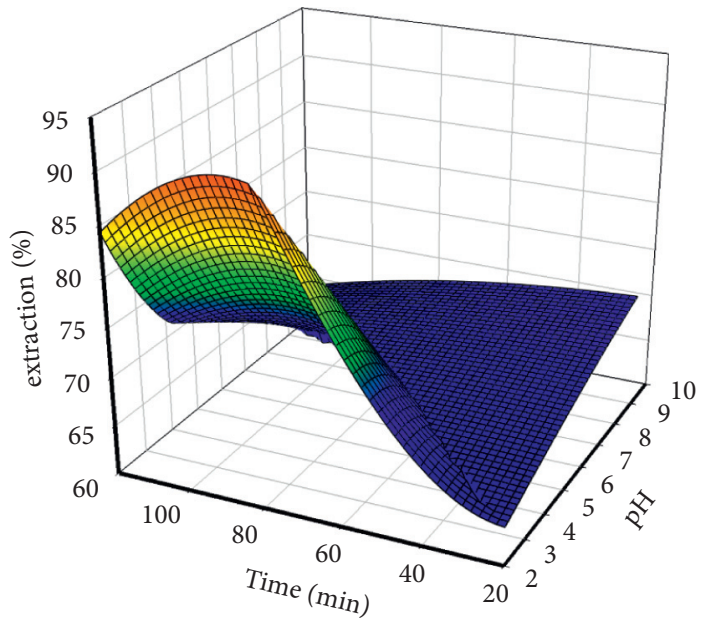

(d)

Figure 7: 3D plots of the interaction influence of solution $\mathrm{pH}$ and contact time on the extraction\% of RY145 by (a) CTAB and (b) SDS, RB222 by (c) CTAB and (d) SDS.

$10^{-5} \mathrm{~mol} / 10 \mathrm{ml}$ and keeping the concentration of surfactant constant. From this figure, it is shown that increasing dye concentration at constant surfactant concentration and producing a fixed number of reverse micelles resulted in a decrease in partition coefficient $(K)$ of RY145 and RB222 dyes. With the increase in the concentration of RY145 and RB222 dyes, the uptake does not take place for this dye in the reverse micelles, leading to an increase in the concentration of dyes in aqueous phase, because the organic phase becomes saturated with dye, and this results agrees with literature [29].

3.3.2. Effect of Surfactant Concentration. The influence of the concentration of CTAB on partition coefficient $(K)$ was studied for RB222 and RY145 from the aqueous phase as shown in Figure 14. From this figure, it is illustrated that, with an increase in the concentration of $\mathrm{CTAB}$ from $9 \times 10^{-3}$ to $9 \times 10^{-2} \mathrm{~mol} / 10 \mathrm{ml}$ ethyl acetate for a given dye concentration, the partition coefficient of both dyes increases due to the presence of force of attraction between opposite charges of dyes molecules and surfactants leading to encapsulation of dye from aqueous layer within reverse micelles [30].

3.4. Separation Kinetics on Dyes Extraction. The kinetics of liquid-liquid extraction process was investigated to explain the relationship between the concentration of surfactants and the separation of dye from aqueous phase. Figures 15 and 16 show that the concentration of dye decreased continuously with time. Consequently, the rate constant was determined for these curves by using first-order kinetics, and it turned out that the decrease in the concentration of ionic dye with time was fitted with first-order model in agreement with literature [31]:

$$
\log [C]=\log \left[C_{0}\right]-\left(\frac{k_{1}}{2.303}\right) t
$$

where $[C] \mathrm{mol} / \mathrm{L}$ is the ionic dye concentration in solution, $k_{1}\left[\mathrm{~min}^{-1}\right]$ is the rate constant of first order, $t$ is the time removal in minute, and $\left[C_{0}\right] \mathrm{mol} / \mathrm{L}$ is the initial ionic 


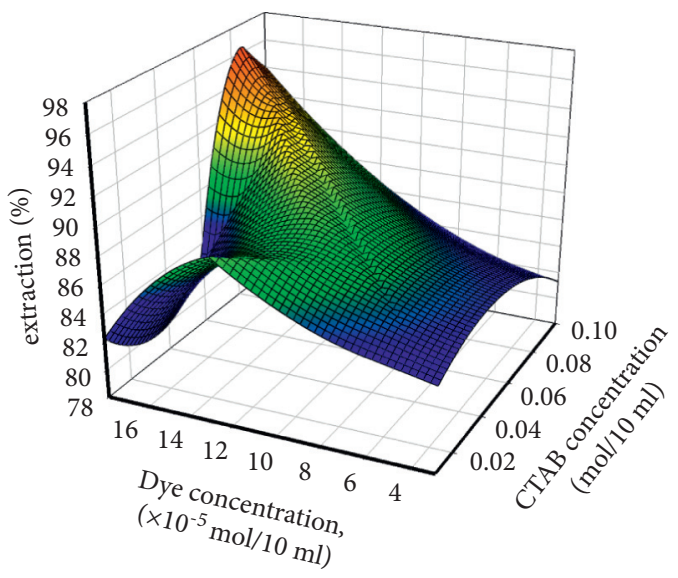

(a)

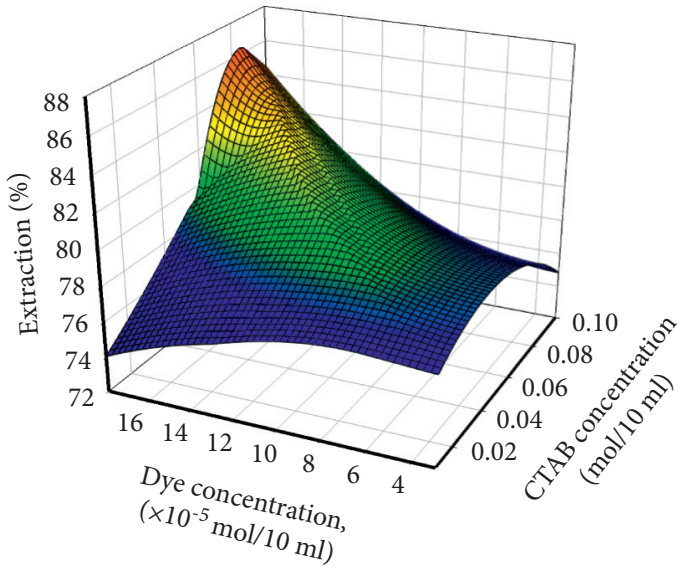

(c)

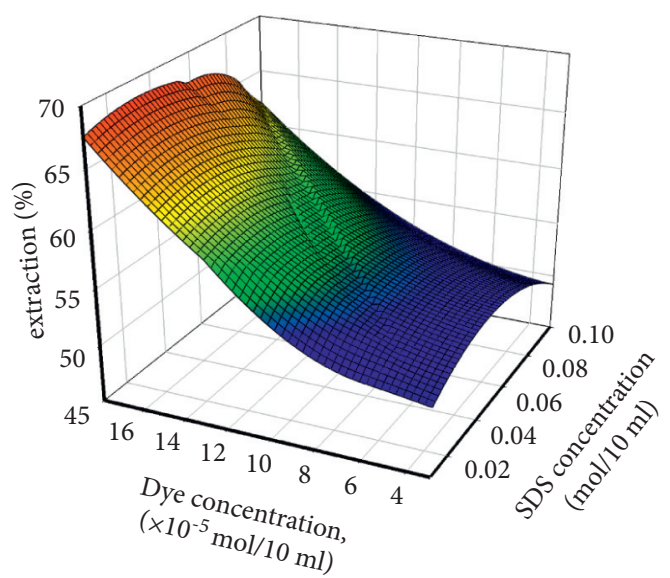

(b)

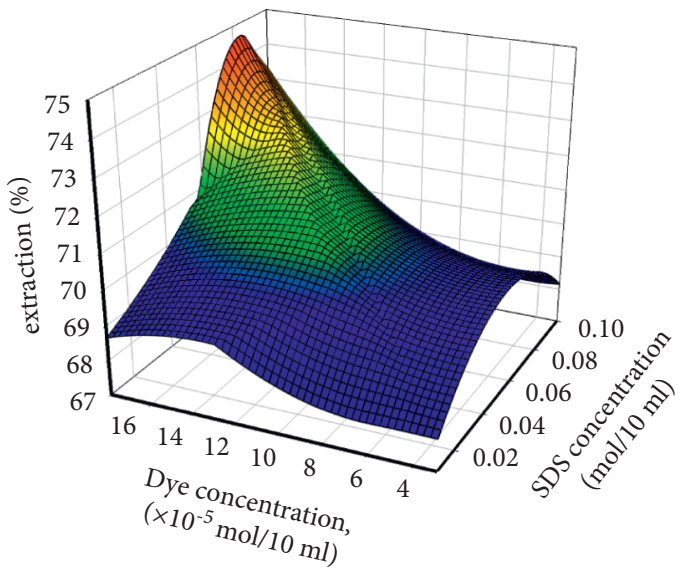

(d)

FIGURE 8: 3D plots of the interaction effect for concentration of surfactant and the initial concentration of dye on the extraction $\%$ of RY145 by (a) CTAB and (b) SDS and RB222 by (c) CTAB and (d) SDS.

dye concentration. The results of a plot of $\log [c]$ against $t$ were obtained by using (6) for RY145 and RB222, and it should give a straight line with a slope $-k_{1} / 2.303$, and $\log$ $\left[C_{0}\right]$ is the intercept. The $k_{1}$ value in separation of rate constant was calculated for the separation of RY145 and RB222 dyes by reverse micelle of surfactants CTAB and SDS at constant temperature $25^{\circ} \mathrm{C}$. The results are summarized in Table 2. Figures 10 and 11 resulted in excellent first-order fitting with a correlation coefficient $\left(R^{2}\right)$ of 0.999 for RY145 and 0.998 for RB222. The half-life time was determined from the obtained rate constants $\left(k_{1}\right)$ using the relationship [18]:

$$
t_{0.5}=\frac{(\ln 2)}{k_{1}},
$$

For the separation phases that correspond to the general water clarification. Obviously, low $t_{0.5}$ value and high removal percentage values mean favorable experimental conditions under which the phases are rapidly separated with efficient water purification. It is possible to express the pseudo-second-order equation based on separation equilibrium in the following form:

$$
\frac{\mathbf{t}}{\mathbf{C}}=\frac{1}{\mathbf{k}_{2} \mathrm{C}_{0}^{2}}+\frac{\mathbf{t}}{\mathrm{C}_{0}},
$$

where $k_{2}\left(\mathrm{~g} \mathrm{mg}^{-1} \mathrm{~min}^{-1}\right)$ is pseudo-second-order rate constant for separation process. To understand the applicability of the models, plot of $t / c$ against $t$ as shown in Figures 17 and 18 gives a relatively straight line and confirms that pseudo-second-order equation is applicable. Pseudosecond-order kinetic $k_{2}$ values and correlation coefficients $R^{2}$ were found and presented in Table 2. From this data, it is illustrated that correlation coefficient in pseudo-secondorder model is lower than pseudo-first-order model and half-life time in pseudo-second-order model is higher than pseudo-first-order model so the pseudo-first-order model are fitted for separation process of RY145 and RB222 from aqueous phase by reverse micelles.

3.5. Stripping Reagent Types for Organic Phase Loaded. Separation of dyes from solvent organic phase for any extraction is very important economically where the organic solvent may be reused other time without change or loss in efficiency. In this study, different inorganic acids or bases 


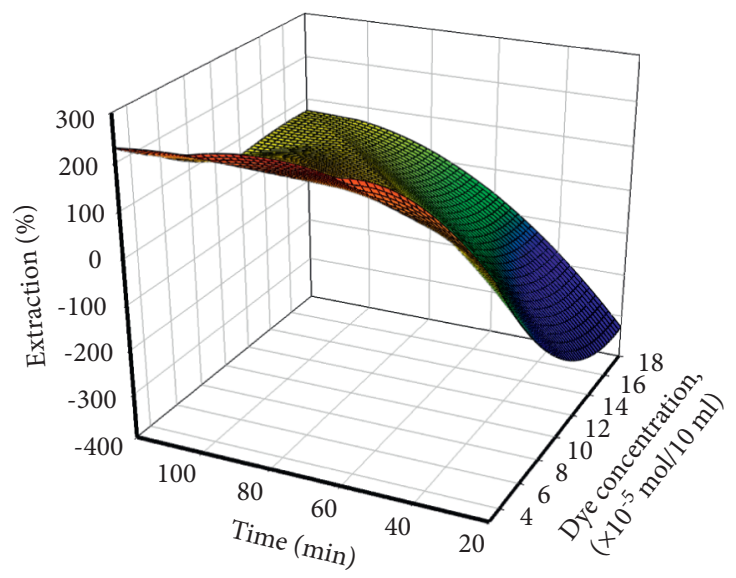

(a)

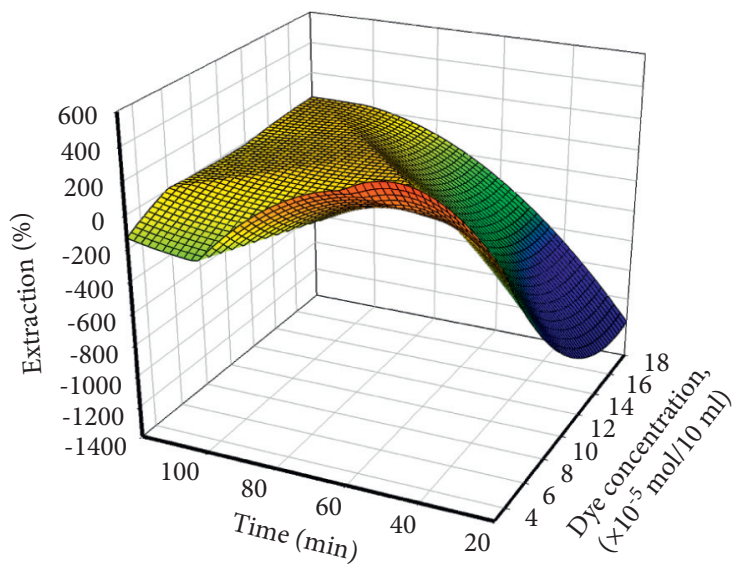

(c)

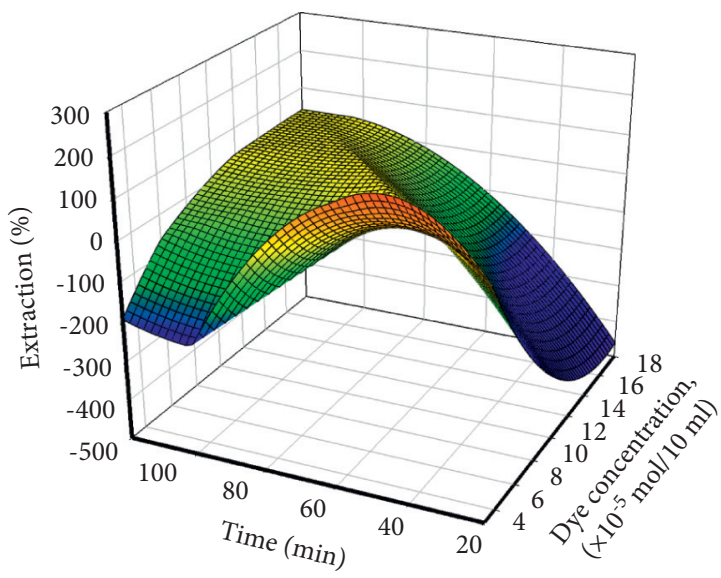

(b)

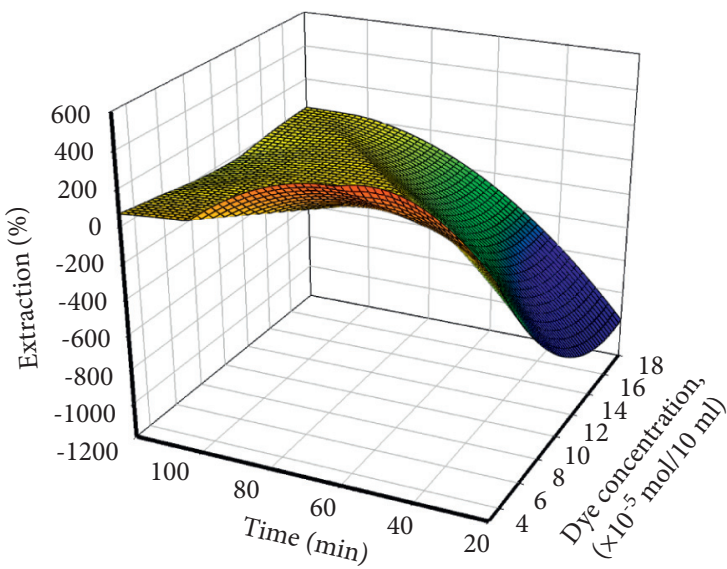

(d)

FIGURE 9: 3D plots of the interaction influence of the time contact with the initial concentration of dye on extraction\% of RY145 by (a) CTAB and (b) SDS and RB222 by (c) CTAB and (d) SDS.

were used as stripping agents, such as hydrochloric acid, sulfuric acid, and sodium hydroxide. Figure 19 indicates the effect of stripping agent on RB222 and RY145 extracted and from this figure, it is shown that, for anionic RY145 and RB222 dyes, the acidic solution gives low extraction efficiency [32]. The results illustrated that the best stripping reagent for RY145 and RB222 is sodium hydroxide.

3.5.1. Concentration of Stripping Agent Effect. In the process of stripping dye from the loaded organic media, stripping concentration of reagent plays an important role, and the results are shown in Figure 20. As stripping agent, sodium hydroxide was used, and its concentration with various molarity ranged from 0.2 to $1.5 \mathrm{M}$. It can be seen from the figure that the efficiency of stripping increased with increase in concentration of $\mathrm{NaOH} .1 \mathrm{M} \mathrm{NaOH}$ solutions were stripped of the higher amount of dye [12-23, 25]. The efficiency of stripping was not improved by further increases in the concentration of $\mathrm{NaOH}$. The $1 \mathrm{M} \mathrm{NaOH}$ solution was selected for further studies.

3.6. Solvent Reusability. The stripped organic solvent is reused to reextract RY145 and RB222 from aqueous solution. When the stripping solvent is reused for dyes and reextracted under the optimized condition, it was found that $93 \%$ of RY 145 and $85 \%$ of RB222 dye were extracted in each run and up to 6 runs of extraction efficiency remained constant. Figure 21 presents the results and shows that the extraction efficiency slightly decreased. This could be caused by the loss of ethyl acetate during dye stripping. Lais et al. [32] also stated that the extraction efficiency decreased with increasing the number of times, and the extractant was recycled when the stripping solvent was reused for methylene blue $(\mathrm{MB}+)$ dye. 


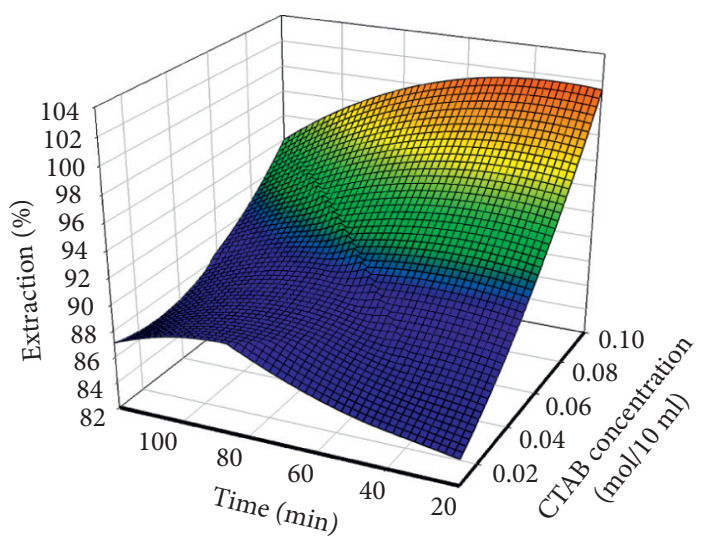

(a)

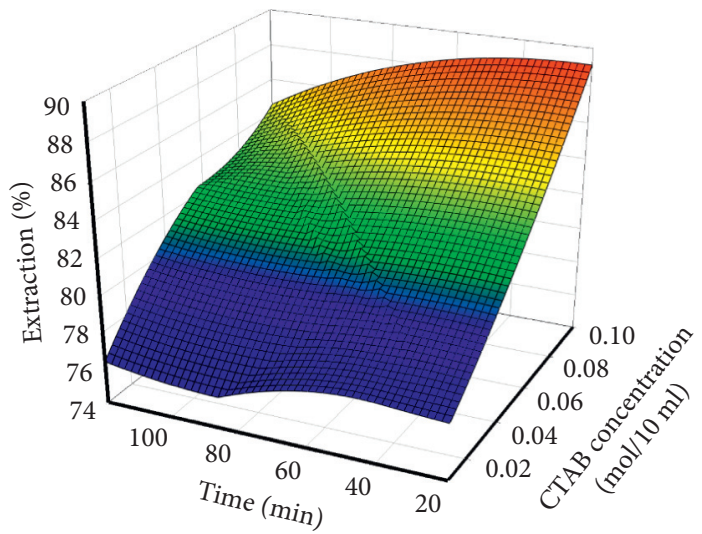

(c)

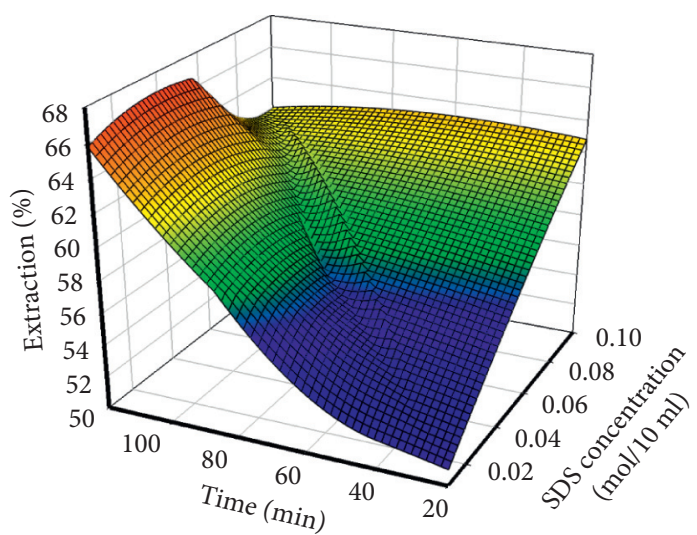

(b)

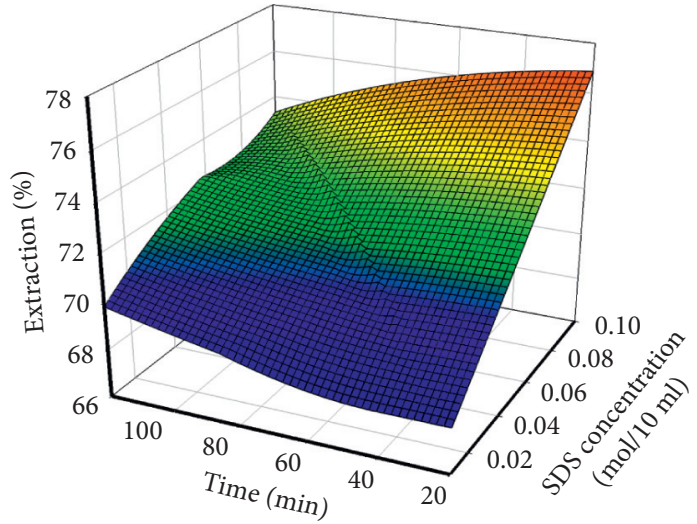

(d)

FIGURE 10: 3D plots of the interaction influence of the contact of time and surfactants concentration on the extraction\% of RY145 by (a) CTAB and (b) SDS and RB222 by (c) CTAB and (d) SDS.

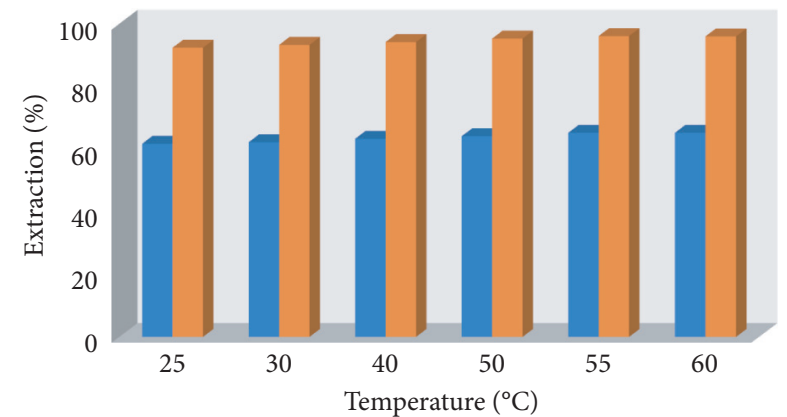

- SDS

- CTAB

FIGURE 11: Effects of the temperature on extraction\% of RY145 at concentration $6 \times 10^{-5} \mathrm{~mol} / 10 \mathrm{ml}$ (contact time: $50 \mathrm{~min}$ ), surfactant concentration $9 \times 10^{-2} \mathrm{~mol} / 10 \mathrm{ml}$, and solution $\mathrm{pH} 7.2$ for CTAB and $\mathrm{pH} 4.2$ for SDS.

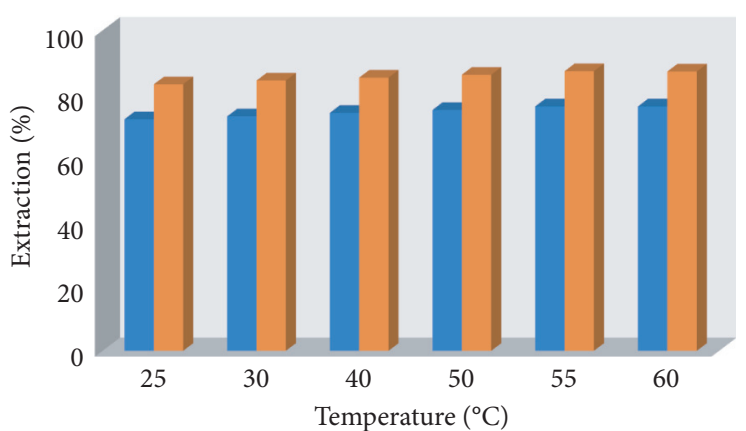

- SDS

- CTAB

FIgURE 12: Effects of the temperature on extraction\% of RB222 at concentration $6 \times 10^{-5} \mathrm{~mol} / 10 \mathrm{ml}$ (contact time: $60 \mathrm{~min}$ ), surfactant concentration $9 \times 10^{-2} \mathrm{~mol} / 10 \mathrm{ml}$, and solution $\mathrm{pH} 7.2$ for CTAB and $\mathrm{pH} 4.2$ for SDS. 


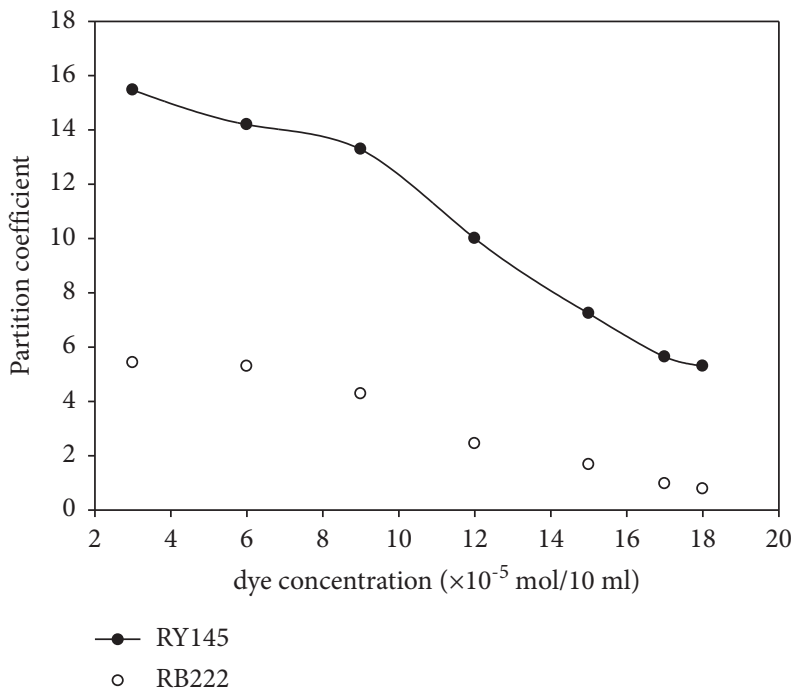

FigURE 13: Partition coefficient (K) by varying the concentration of RY145 and RB222.

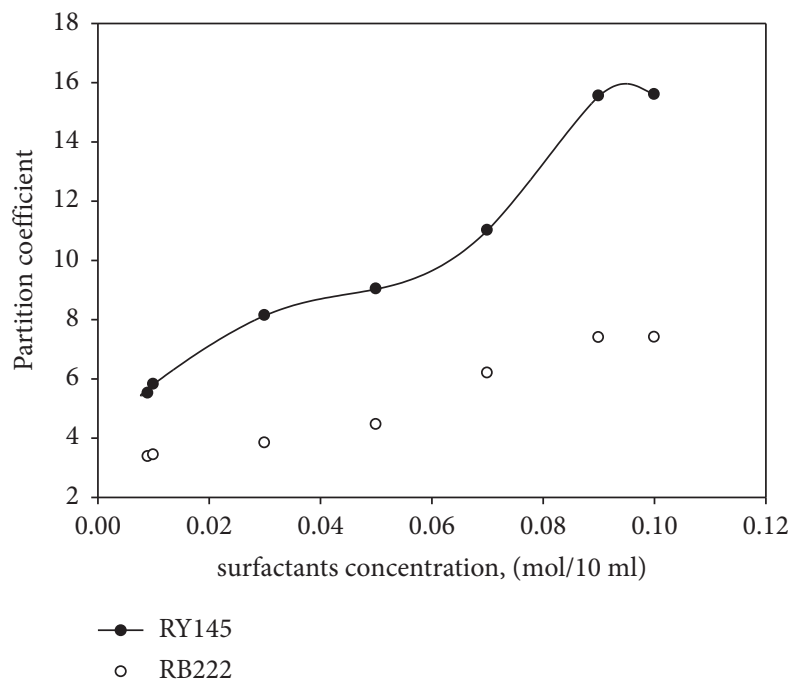

Figure 14: Partition coefficient $(K)$ for RY145 and RB222 by varying the concentration of the surfactant.

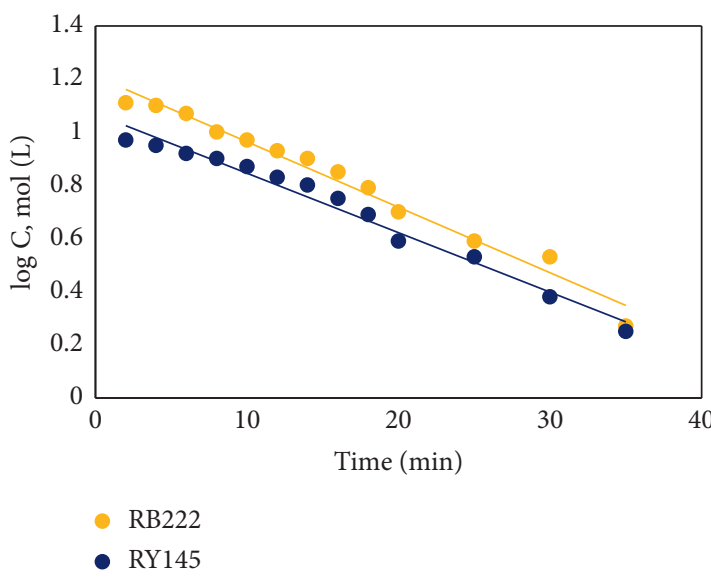

FIgURE 15: First-order kinetic for extraction of RY145 and RB222 dyes at concentration $6 \times 10^{-5} \mathrm{~mol} / 10 \mathrm{ml}$ by $\mathrm{CTAB}$ surfactant concentration $9 \times 10^{-2} \mathrm{~mol} / 10 \mathrm{ml}$.

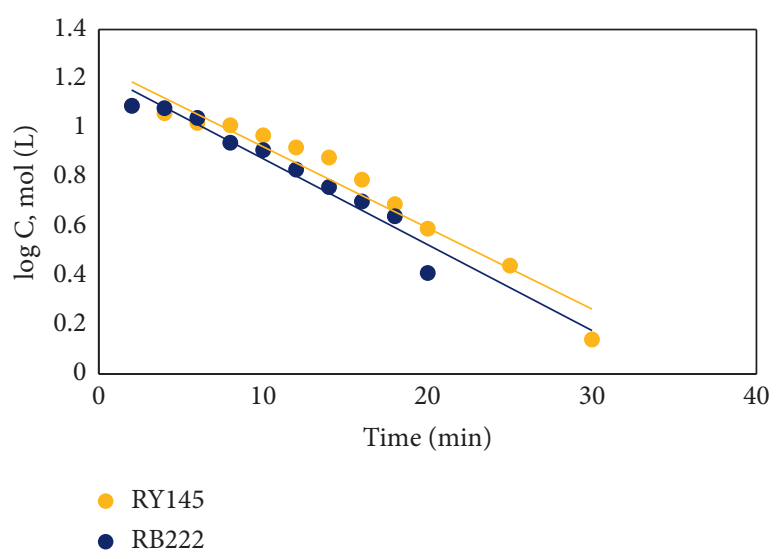

FIgURE 16: First-order kinetic for extraction of RY145 and RB222 dyes at concentration $6 \times 10^{-5} \mathrm{~mol} / 10 \mathrm{ml}$ by SDS surfactant concentration $9 \times 10^{-2} \mathrm{~mol} / 10 \mathrm{ml}$. 
TABLE 2: Summarizing the constants of kinetics model and the correlation coefficients for RY145 and RB222 by SDS and CTAB surfactants.

\begin{tabular}{|c|c|c|c|c|c|c|}
\hline \multirow{3}{*}{ Surfactants } & \multicolumn{6}{|c|}{ Pseudo-first-order model } \\
\hline & \multicolumn{3}{|c|}{ RY145 } & \multicolumn{3}{|c|}{ RB222 } \\
\hline & $k_{1} \min ^{-1}$. & $R^{2}$ & $t_{0.5} \min$ & $k_{1} \min ^{-1}$ & $R^{2}$ & $t_{0.5} \min$ \\
\hline CTAB & 0.0804 & 0.999 & 8.62 & 0.0555 & 0.9980 & 12.5 \\
\hline SDS & 0.0613 & 0.9772 & 11.31 & 0.0760 & 0.9733 & 9.11 \\
\hline \multicolumn{7}{|c|}{ Pseudo-second-order model } \\
\hline & & 145 & & & 22 & \\
\hline & $k_{2} \mathrm{~g} \mathrm{mg}^{-1} \mathrm{~min}^{-1}$ & $R^{2}$ & $t_{0.5} \min$ & $k_{2} \mathrm{~g} \mathrm{mg}^{-1} \min ^{-1}$ & $R^{2}$ & $t_{0.5} \min$ \\
\hline CTAB & 0.0029 & 0.9871 & 15.52 & 0.0039 & 0.9571 & 17.54 \\
\hline SDS & 0.0029 & 0.9461 & 15.52 & 0.0014 & 0.9441 & 32.14 \\
\hline
\end{tabular}

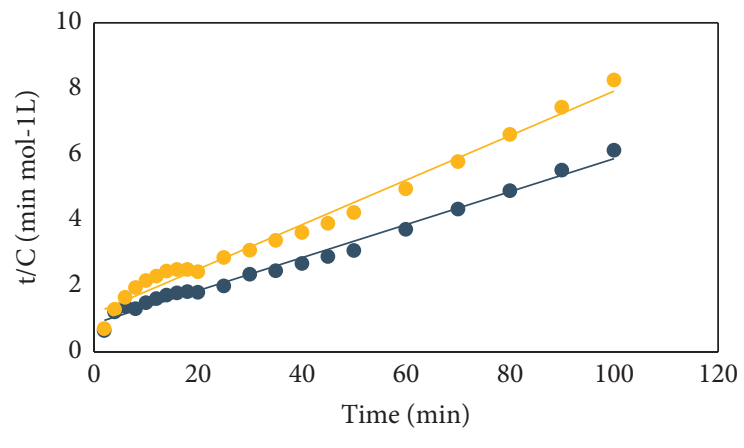

- RB222

RY145

FIgURE 17: Pseudo-second-order kinetic model for extraction of RY145 and RB222 dyes at concentration $6 \times 10^{-5} \mathrm{~mol} / 10 \mathrm{ml}$ by SDS surfactant concentration $9 \times 10^{-2} \mathrm{~mol} / 10 \mathrm{ml}$.

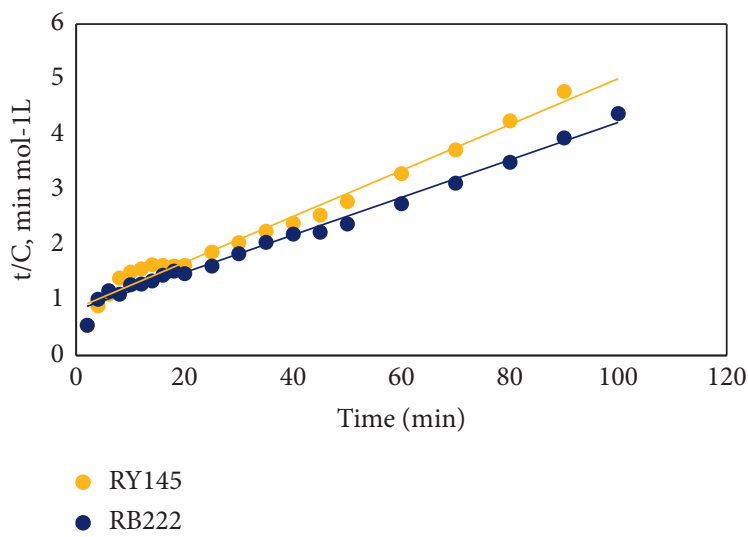

FIgURe 18: Pseudo-second-order kinetic model for extraction of RY145 and RB222 dyes at concentration $6 \times 10^{-5}$ mol/10 ml by CTAB surfactant concentration $9 \times 10^{-2} \mathrm{~mol} / 10 \mathrm{ml}$. 


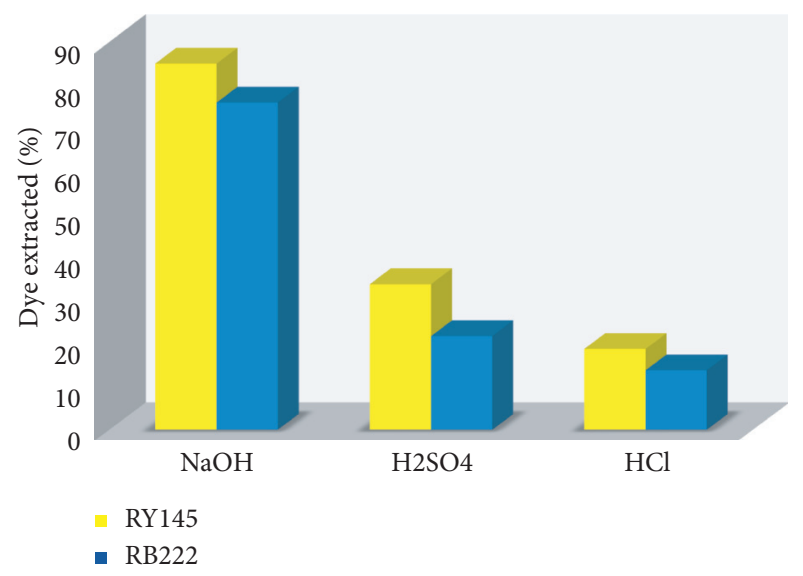

FIGURE 19: Effect of stripping reagents on extracted RB222 and RY145.

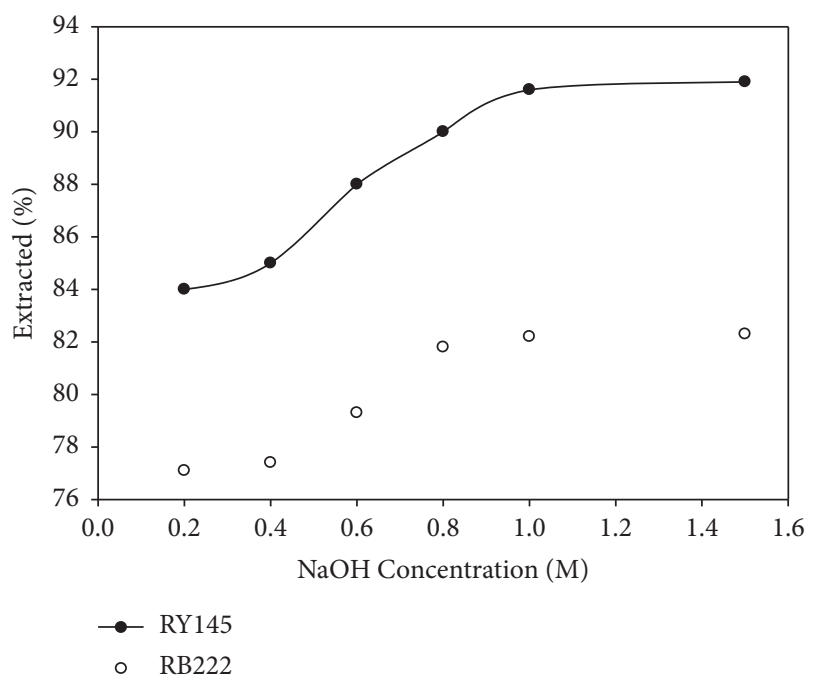

FIGURE 20: Effect of the concentration of stripping agent (at optimum conditions, initial dye concentration $6 \times 10^{-5} \mathrm{~mol} / 10 \mathrm{ml}$, solution $\mathrm{pH} 7.2$ at $25^{\circ} \mathrm{C}$ ).

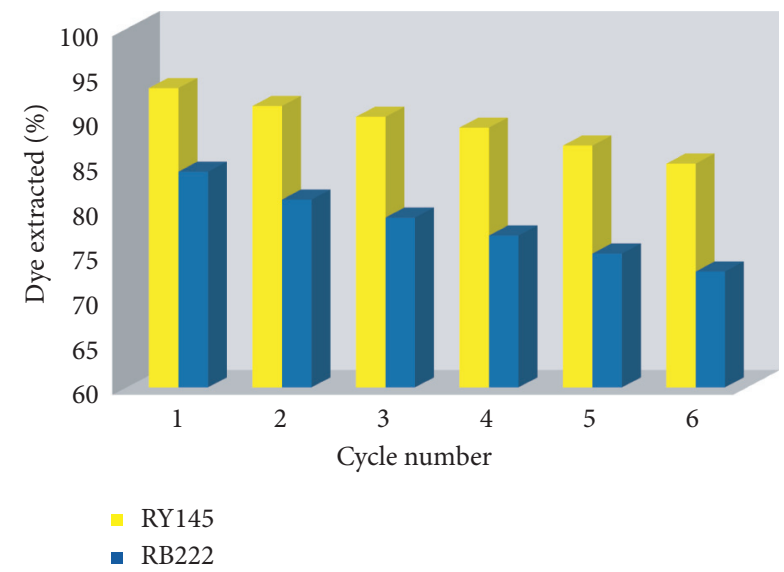

FIGURE 21: Efficiency of ethyl acetate recovery and loading capacity of CTAB (experimental conditions, initial dye concentration $6 \times 10^{-5} \mathrm{~mol} / 10 \mathrm{ml}$, solution $\mathrm{pH} 7.2$ at $25^{\circ} \mathrm{C}$, equilibration time $60 \mathrm{~min})$.

\section{Conclusion}

Although many people throughout the world lack access to clean and hygienic drinking water, it is considered one of the most vital commodities and is considered the base of power and life for human survival. Population growth, rapid industrialization, and widespread usage of nonrenewable resources have all wreaked havoc on the ecosystem. Discharges and effluents from various factories as a result of human activity harm and damage our natural resources.

So, we derived a new protocol in the present study for dye separation from water, described as follows:

(i) Reverse micelles of anionic and cationic surfactants were used in the process of solvent extraction for the separation of anionic dyes.

(ii) The separation mechanism has been shown to be primarily by electrostatic interactions between surfactant ions and dye ions.

(iii) Dye extraction of RY145 and RB222 from aqueous phase and partition coefficient increase with the increase in surfactant concentration, since the number of reverse micelles is expected to increase linearly with the increasing concentration of surfactant, which in turn increases the number of dye molecules that can be encapsulated.

(iv) The surfactant used in this method can be separated and the fit concentration for $\mathrm{NaOH}$ as stripping agent is $1 \mathrm{M}$ for separation of dyes from solvent organic phase for any extraction; also the solvent can be reused for further extraction up to 6 times, so this method to nature is ecofriendly.

We concluded that the optimized conditions for the maximum extraction of dyes were noticed at $\mathrm{pH}$ ( 4 and $7 \pm 0.2$ ) by SDS and CTAB, respectively, for both dyes, extractant concentration of $9 \times 10^{-2} \mathrm{~mol} / 10 \mathrm{ml}$ ethyl acetate, $1 \mathrm{M} \mathrm{NaOH}$ stripping reagent, time of extraction $60 \mathrm{~min}$, and the initial concentration of dye $6 \times 10^{-2} \mathrm{~mol} /$ $10 \mathrm{ml}$. It was found that more than $62 \%$ and $72 \%$ of RY145 and RB222, respectively, were removed by SDS surfactant and more than $94 \%$ and $85 \%$ of RY145 and RB222, respectively, were removed by CTAB surfactant at $25^{\circ} \mathrm{C}$.

\section{Data Availability}

The data used to support the findings of this study are available from the corresponding author upon request.

\section{Ethical Approval}

This article does not contain any studies with human participants or animals performed by the authors.

\section{Conflicts of Interest}

The authors declare that they have no conflicts of interest. 


\section{Acknowledgments}

The authors would like to thank the Faculty of Science, Department of Chemistry, Al-Azhar University, for chemicals supply.

\section{References}

[1] M. K. Purkait, S. Das Gupta, and S. De, "Removal of dye from wastewater using micellar-enhanced-ultrafiltration and recovery of surfactant," Separation and Purification Technology, vol. 37, no. 1, 2004.

[2] A. Idris, M. Sani, A. Wasila, and A. Adamu, "Adsorption isotherms and kinetic studies of congo-red removal from wastewater using activated carbon prepared from jujube seed," Journal of Analytical Chemistry, vol. 11, pp. 47-59, 2020.

[3] S. Gunatilake, "Methods of removing heavy metals from industrial wastewater," Journal of Multidiciplinary Engineering Science Studies, vol. 1, 2015.

[4] W. Tiecheng, W. Qi, S. Heng et al., "A green strategy for simultaneous $\mathrm{Cu}(\mathrm{II})$-EDTA decomplexation and $\mathrm{Cu}$ precipitation from water by bicarbonate-activated hydrogen peroxide/precipitation," Chemical Engineering Journal, vol. 370, pp. 1298-1309, 2019.

[5] G. Rashi, G. Kanika, J. Pratiksha, and K. Om, “Adsorptive removal and photocatalytic degradation of organic pollutants using metal oxides and their composites: a comprehensive review," Advances in Colloid and Interface Science, vol. 272, pp. 10-20, 2019.

[6] Y. Zhang, B. Wu, H. Xu et al., "Nanomaterials-enabled water and wastewater treatment," NanoImpact, vol. 3-4, pp. 22-39, 2016.

[7] R. K. Thines, N. M. Mubarak, S. Nizamuddin, J. N. Sahu, E. C. Abdullah, and P. Ganesan, "Application potential of carbon nanomaterials in water and wastewater treatment: a review," Journal of the Taiwan Institute of Chemical Engineers, vol. 72, pp. 116-133, 2017.

[8] K. Gupta and O. P. Khatri, "Fast and efficient adsorptive removal of organic dyes and active pharmaceutical ingredient by microporous carbon: effect of molecular size and charge," Chemical Engineering Journal, vol. 378, pp. 1-12, 2019.

[9] M. S. Cristina, S. F. Zoraida, T. P. Esther, and J. S. Jose', "Application of new approaches to liquid-phase microextraction for the determination of emerging pollutants," Trends in Analytical Chemistry, vol. 30, pp. 731-748, 2011.

[10] S. Nishihama, T. Hirai, and I. Komasawa, "Review of advanced liquid-liquid extraction systems for the separation of metal ions by a combination of conversion of the metal species with chemical reaction," Industrial \& Engineering Chemistry Research, vol. 40, no. 14, pp. 3085-3091, 2001.

[11] A. C. Ueda, L. H. De Oliveira, N. Hioka, and M. Aznar, "Liquid-liquid extraction of basic yellow 28, basic blue 41, and basic red 46 dyes from aqueous solutions with reverse micelles," Journal of Chemical \& Engineering Data, vol. 56, no. 3, pp. 652-657, 2011.

[12] L. Bukman, A. L. Tessaro, N. R. C. Fernandes-Machado, W. Caetano, and N. Hioka, "Treatment of wastewater contaminated with ionic dyes: liquid-liquid extraction induced by reversed micelle followed by photodegradation," Separation and Purification Technology, vol. 189, pp. 162-169, 2017.

[13] A. Dukhin and S. Parlia, "Ions, ion pairs and inverse micelles in non-polar media," Current Opinion in Colloid \& Interface Science, vol. 18, no. 2, pp. 93-115, 2013.
[14] X. Sun and N. Bandara, "Applications of reverse micelles technique in food science: a comprehensive review," Trends in Food Science \& Technology, vol. 91, pp. 106-115, 2019.

[15] W. Al-Soufi, L. Piñeiro, and M. Novo, "A model for monomer and micellar concentrations in surfactant solutions: application to conductivity, NMR, diffusion, and surface tension data," Journal of Colloid and Interface Science, vol. 370, no. 1, pp. 102-110, 2012.

[16] K. Manna and A. K. Panda, "Physicochemical studies on the interfacial and micellization behavior of CTAB in aqueous polyethylene glycol media," Journal of Surfactants and Detergents, vol. 14, no. 4, pp. 563-576, 2011.

[17] P. M. Juan and M. Martín, "Thermodynamics of sodium dodecyl sulfate (SDS) micellization: an undergraduate laboratory experiment," Chemical Engineering Journal, vol. 88, pp. 629-633, 2011.

[18] Y. Shixiong, S. Gang, and D. Fangyin, "Removal and separation of mixed ionic dyes by solvent extraction," Textile Research Journal, vol. 1, pp. 1-9, 2017.

[19] L. M. J. Sprakel and B. Schuur, "Solvent developments for liquidliquid extraction of carboxylic acids in perspective," Separation and Purification Technology, vol. 211, pp. 935-957, 2019.

[20] S. P. Jeevan Kumar, G. Vijay Kumar, A. Dash, P. Scholz, and R. Banerjee, "Sustainable green solvents and techniques for lipid extraction from microalgae: a review," Algal Research, vol. 21, pp. 138-147, 2017.

[21] X. Peng, X.-Z. Yuan, G.-M. Zeng et al., "Extraction and purification of laccase by employing a novel rhamnolipid reversed micellar system," Process Biochemistry, vol. 47, no. 5, pp. 742-748, 2012.

[22] A. Rahdar, M. Aliahmad, A. M. Kor, and D. Sahoo, "Probing the reverse micelle environment with a cationic dye by varying oil and water content of micelles," Spectrochimica Acta Part A: Molecular and Biomolecular Spectroscopy, vol. 210, pp. 165170, 2019.

[23] H. Boualem, "Temperature effect on the nanostructure of SDS Micelles in water," Journal of Research of the National Institute of Standards and Technology, vol. 118, pp. 151-167, 2013.

[24] P. Pandit and S. Basu, "Removal of ionic dyes from water by solvent extraction using reverse micelles," Environmental Science \& Technology, vol. 38, no. 8, pp. 2435-2442, 2004.

[25] K. M. Charanjeet, K. Satindar, D. Kamal, K. Amandeep, and C. Anjali, "Extraction of hazardous direct green b dye with application of reverse micelles of gemini surfactants," International Journal of Latest Technology in Engineering, Management \& Applied Science (IJLTEMAS), vol. 5, pp. 2278-2540, 2016.

[26] K. Narinderjit, S. Sangeeta, and K. Ekta, "Binary cationic dyescounter ion extraction by reverse micelles," Tenside Surfactants Detergents, vol. 57, pp. 31-39, 2020.

[27] C. K. Mangat and S. Kaur, "Efficient removal and separation of anionic dyes from aqueous medium by the application of reverse micelles of cationic surfactants," Desalination and Water Treatment, vol. 52, no. 19-21, pp. 3555-3563, 2014.

[28] B. Mandal, S. Ghosh, and S. P. Moulik, "Interaction between a bio-tolerable amino-acid based amphiphile (N-dodecanoylsarcosinate, SDDS) and modified cationic polymers, hydroxyethylcelluloses (JR 400, and LM 200) in isopropanolwater medium," Colloids and Surfaces A: Physicochemical and Engineering Aspects, vol. 566, pp. 156-165, 2019.

[29] R. Tuteja, N. Kaushik, C. P. Kaushik, and J. K. Sharma, "Recovery of reactive (triazine) dyes from textile effluent by solvent extraction process," Chemistry: An Asian Journal, vol. 22, pp. 539-545, 2010. 
[30] P. Ajmal Koya, K. Kabir-ud-Din, and K. Ismail, "Micellization and thermodynamic parameters of butanediyl-1,4-bis(tetradecyldimethylammonium bromide) gemini surfactant at different temperatures: effect of the addition of 2-methoxyethanol," Journal of Solution Chemistry, vol. 41, no. 8, pp. 1271-1281, 2012.

[31] G. Yusuke, N. Yuya, and M. Keisuke, "Foam separation of dyes using anionic, cationic, and amphoteric surfactants," Journal of Oleo Science, vol. 69, pp. 549-555, 2020.

[32] B. Lais, R. D. Vagner, R. C. Nadia, C. Wilker, R. B. Vagner, and H. Noboru, "Reverse micellar extraction of dyes based on fatty acids and recoverable organic solvents," Separation and Purification Technology, vol. 242, pp. 1-8, 2020. 